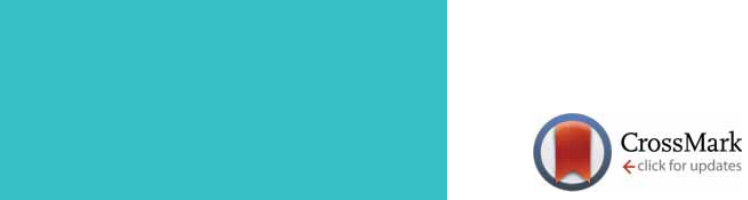

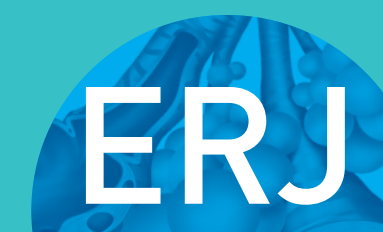

open research
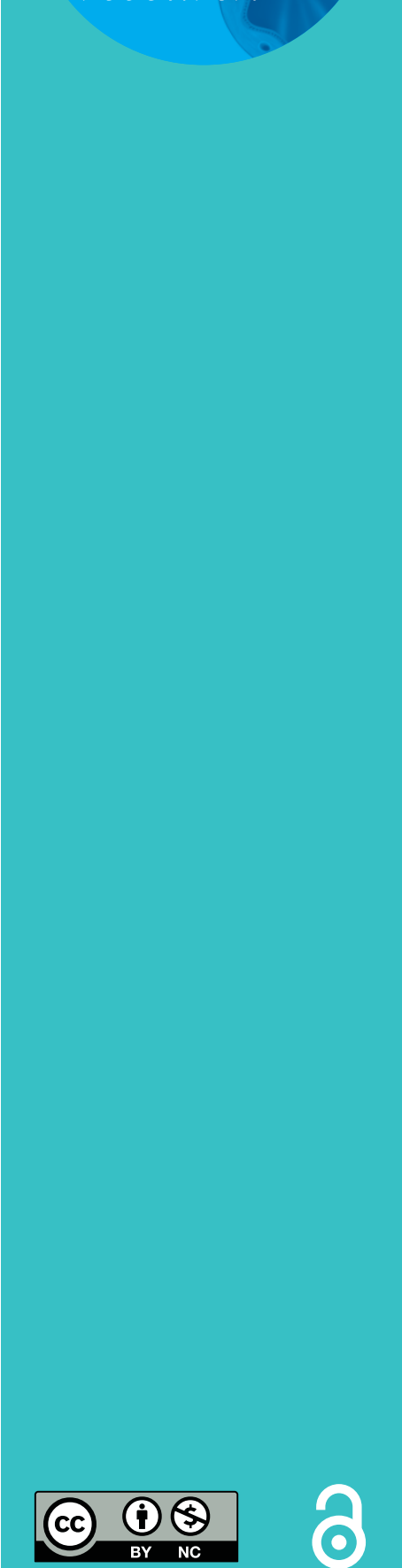

\section{VAC chemotherapy with valproic acid for refractory/relapsing small cell lung cancer: a phase II study}

\author{
Thierry Berghmans ${ }^{1}$, Jean-Jacques Lafitte ${ }^{2}$, Arnaud Scherpereel ${ }^{2}$, \\ Lieveke Ameye ${ }^{3}$, Marianne Paesmans ${ }^{3}$, Anne-Pascale Meert ${ }^{1}$, Benoit Colinet ${ }^{4}$, \\ Christian Tulippe ${ }^{5}$, Luc Willems ${ }^{6}$, Nathalie Leclercq ${ }^{1}$ and Jean-Paul Sculier ${ }^{1}$, \\ for the European Lung Cancer Working Party
}

Affiliations: 'Department of Intensive Care Unit and Thoracic Oncology, Institut Jules Bordet, Centre des Tumeurs de l'Université Libre de Bruxelles (ULB), Brussels, Belgium. ${ }^{2}$ Department of Pulmonary and Thoracic Oncology, CHRU de Lille and University of Lille II, France. ${ }^{3}$ Data Centre, Institut Jules Bordet, Centre des Tumeurs de l'ULB, Brussels, Belgium. ${ }^{4}$ Department of Pneumology, Grand Hôpital de Charleroi, Gilly, Belgium. ${ }^{5}$ Department of Pneumology, $\mathrm{CH}$ Mouscron, Mouscron, Belgium. ${ }^{6}$ Gembloux Agro-Bio Tech and Interdisciplinary Cluster for Applied Genoproteomics (GIGA), University of Liège, Gembloux, Belgium.

Correspondence: Thierry Berghmans, Institut Jules Bordet, Rue Héger-Bordet 1, 1000 Bruxelles, Belgium. E-mail: thierry.berghmansabordet.be

ABSTRACT Salvage chemotherapy (CT) for relapsing or refractory small cell lung cancer (SCLC) remains disappointing. In vitro experiments showed that valproic acid increases apoptosis of SCLC cell lines exposed to doxorubicin, vindesine and bis(2-chloroethyl)amine. The primary objective of this phase II study was to determine whether epigenetic modulation with valproic acid in addition to a doxorubicin, vindesine and cyclophosphamide (VAC) regimen improves 6-month progression-free survival (PFS).

Patients with pathologically proven SCLC refractory to prior platinum derivatives and etoposide were eligible. After central registration, patients received VAC plus daily oral valproic acid.

64 patients were registered, of whom six were ineligible. Seven patients did not receive any CT, leaving 51 patients assessable for the primary end-point. The objective response rate was $19.6 \%$. Median PFS was 2.8 months (95\% CI 2.5-3.6 months) and 6-month PFS was 6\%. Median survival time was 5.9 months (95\% CI 4.7-7.5 months). Toxicity was mainly haematological, with $88 \%$ and $26 \%$ grade $3-4$ neutropenia and thrombopenia, respectively.

Despite an interesting response rate, the addition of valproic acid to VAC did not translate into adequate PFS in relapsing SCLC or SCLC refractory to platinum-etoposide.

@ERSpublications

Epigenetic modulation with valproic acid does not improve CT efficacy in refractory SCLC after platinum-etoposide http://ow.ly/R0rBt

Received: May 222015 | Accepted after revision: Aug 092015

Clinical trial: This study is registered with EudraCT with identifier number 2008-004278-41 and www.clinicaltrials.gov with identifier number NCT00759824.

Conflict of interest: None declared.

Copyright $\odot$ ERS 2015. This article is open access and distributed under the terms of the Creative Commons Attribution Non-Commercial Licence 4.0. 


\section{Introduction}

Small cell lung cancer (SCLC) accounts for $<15 \%$ of all lung cancer [1]. SCLC is usually initially chemotherapy (CT)-sensitive and first-line treatment is associated with high objective response rates (RRs) $(45-75 \%)$. As observed in meta-analyses $[2,3]$, platinum derivatives and etoposide are the most active drugs and are currently considered as the standard for first-line treatment. However, in most of the patients, SCLC will recur and these patients will be candidates for second-line CT. A randomised trial [4] and systematic reviews $[5,6]$ suggested that better survival is observed in patients receiving second- or third-line treatment in comparison with supportive care only. Nevertheless, the efficacy of salvage CT depends on the response to first-line CT and the time interval before recurrence, with nonresponding patients (refractory) or those with a delay $<3$ months (resistant) having a poorer prognosis in comparison with those with sensitive tumours (objective response with a delay $>3$ months before recurrence), whatever the RR ( $\mathrm{p}=0.0001)$ or median survival time (MST) ( $\mathrm{p}=0.0035)$ [7].

There have been few phase III trials to determine the best CT regimen for relapsed SCLC, mainly testing VAC (vincristine, doxorubicin and cyclophosphamide) and topotecan. In a phase III trial, vON PAWEL et al. [8] compared VAC to oral topotecan in patients relapsing $\geqslant 60$ days after first-line CT. RR and MST were $18.3 \%$ and 24.7 weeks and $24.3 \%$ and 25 weeks, respectively, without any statistically significant difference. More grade 4 thrombocytopenia $(p<0.001)$ and grade $3 / 4$ anaemia $(p<0.001)$ occurred in the topotecan arm. Two other randomised trials compared intravenous to oral topotecan $[9,10]$. No significant differences were found for RR or MST. Haematological toxicity was important in both arms. Based on these data showing similar efficacy but increased toxicity with topotecan, VAC appears an adequate salvage CT.

As the results of second-line CT remain disappointing, original therapeutic concepts have to be considered. In a previous phase II study performed in malignant mesothelioma [11], we showed that adding valproic acid, a histone deacetylase (HDAC) inhibitor, to CT allowed an increase in RR in comparison with historical controls. As lysine deacetylase inhibitor, valproic acid decondenses chromatin and induces gene transcription. Since valproic acid has been widely used for treatment of epilepsy, its pharmacology and toxicology have been very well characterised, including potential interactions with conventional CT. In addition, we demonstrated a synergistic effect on the apoptotic rate in four SCLC cell lines by adding valproic acid to vindesine, doxorubicin and 2-chloroethylamine (the active cyclophosphamide metabolite) (data presented in the associated article by Hubaux et al. [12]).

The primary objective of this phase II study was to determine whether epigenetic modulation with valproic acid in addition to a VAC regimen can improve 6-month progression-free survival (PFS).

\section{Material and methods}

This phase II study was an academic trial (EudraCT identifier number 2008-004278-41). The protocol is available on from www.elcwp.org and www.clinicaltrials.gov (identifier number NCT00759824). The study was approved by all ethical committees of the participating institutions as well as the national representatives.

To be eligible, patients had to fulfil the following eligibility criteria: histological or cytological SCLC diagnosis; SCLC refractory to a prior CT regimen including platinum derivatives (cisplatin or carboplatin) and etoposide, either primary refractory (immediate progression or recurrence $<3$ months after the end of previous CT) or secondary refractory (sensitive patients to platinum plus etoposide in first-line, progressing or recurring $<3$ months after reintroduction of the same CT regimen); at least one evaluable or measurable lesion; adequate haematological (neutrophils $>2000 \mathrm{~mm}^{-3}$, platelets $>100000 \mathrm{~mm}^{-3}$ ), hepatic (serum bilirubin $<1.5 \mathrm{mg}$ per $100 \mathrm{~mL}$ and/or transaminases more than twice the normal range) and renal (serum creatinine $<1.5 \mathrm{mg}$ per $100 \mathrm{~mL}$ ) function; availability for participating in the detailed follow-up of the protocol; and having provided signed informed consent. Criteria for ineligibility were: previous treatment with anthracycline, vinca-alkaloid derivatives or cyclophosphamide; performance status $<60$ on the Karnofsky scale; history of prior malignant tumour, except nonmelanoma skin cancer, in situ carcinoma of the cervix or of the bladder, or cured malignant tumour ( $>5$-year disease free interval); history of HIV infection; abnormal coagulation tests (activated partial thromboplastin time (PTT), PTT or prothrombin time) and/or decreased fibrinogen; recent myocardial infarction $(<3$ months prior to date of diagnosis), congestive cardiac failure (ejection fraction of the left ventricle $<50 \%$ ) or uncontrolled cardiac arrhythmia; uncontrolled infectious disease; active epilepsy needing a specific treatment; concomitant treatment with monoamine oxidase inhibitors, carbamazepine, mefloquine, phenobarbital, primidone (desoxyphenobarbital), phenytoin, lamotrigine or zidovudine; pregnancy or refusal to use active contraception; known allergy to valproic acid and/or doxorubicin, cyclophosphamide or vindesine; and serious medical or psychological factors that might have prevented adherence to the treatment schedule.

Initial work-up consisted of history and physical examination including weight, height and surface area measurement, recording of performance status, complete blood sampling, and ECG with isotopic or 
echographic left ventricular fraction assessment. Tumour assessment included chest radiography and computed tomography, computed tomography or echography of the liver and adrenal glands, brain magnetic resonance imaging or computed tomography, and bone isotopic scintigraphy (with radiography of suspected lesions). If positron emission tomography was performed, bone isotopic scintigraphy could be avoided and abdominal computed tomography or echography were performed in the case of positive lesions. Blood counts were repeated at least once between cycles. Complete blood sampling including valproic acid dosage, chest radiography, ECG, and physical examination were performed before each new course. A complete evaluation including the same tests as in the initial work-up was performed every three cycles. During follow-up, patients had to be seen every 2 months with a clinical examination, biology and chest radiography.

\section{Treatment plan}

After central registration at the European Lung Cancer Working Party (ELCWP) data centre, eligible patients received doxorubicin $\left(45 \mathrm{mg} \cdot \mathrm{m}^{-2}\right.$ on day 1$)$, cyclophosphamide $\left(1 \mathrm{~g} \cdot \mathrm{m}^{-2}\right.$ on day 1 ) and vindesine $\left(3 \mathrm{mg} \cdot \mathrm{m}^{-2}\right.$ on day 1$)$ every 3 weeks. The next course could be given after haematological recovery (neutrophils $>1500 \mathrm{~mm}^{-3}$ and platelet count $>100000 \mathrm{~mm}^{-3}$ ). If myelosuppression persisted at day 36 , the patient went off treatment. Valproic acid was administered orally at a dose of $20-30 \mathrm{mg} \cdot \mathrm{kg}^{-1} \cdot \mathrm{day}^{-1}$ during meals in order to obtain a serum concentration in the range of the recommended values for the treatment of epilepsy (50-100 $\left.\mu \mathrm{g} \cdot \mathrm{mL}^{-1}\right)$. The administration of valproic acid began 1 week before first cycle of VAC and continued during the whole treatment. Response was assessed after three courses and responders pursued CT until the best response was achieved, there was unacceptable toxicity or the cumulative dose of doxorubicin exceeded $500 \mathrm{mg} \cdot \mathrm{m}^{-2}$. At progression, the choice of treatment was left at the discretion of the treating physicians.

The following dose adaptations were proposed during treatment. If during the previous course, the nadir counts of neutrophils and/or platelets was $<500$ or $<25000 \mathrm{~mm}^{-3}$, respectively, the doses of doxorubicin, cyclophosphamide and vindesine were reduced to $75 \%$ for the next course. In the case of asymptomatic or transient cardiac dysrhythmias, or those not requiring therapy (World Health Organization (WHO) grade 1), no change in doxorubicin administration was needed. In the case of WHO grade 3 or 4 toxicity, the treatment had to be stopped. In cases of WHO grade 2 toxicity, treatment continuation was discussed on a case-by-case basis. Treatment was stopped in cases of left ventricular ejection fraction (LVEF) reduction $>10 \%$ from the baseline or a reduction below the absolute value of $50 \%$. In cases of WHO grade $>2$ peripheral polyneuropathy or constipation, vindesine was stopped. In case of stomatitis of WHO grade $\geqslant 3$, the doses of doxorubicin, cyclophosphamide and vindesine were reduced at $75 \%$ for next course. In cases of skin toxicity WHO grade $\geqslant 2$ attributable to valproic acid, it was stopped and VAC continued without dose adaptation. In cases of any other grade $\geqslant 3$ WHO toxicity, if doxorubicin, cyclophosphamide and/or vindesine was responsible, the treatment was stopped, except for alopecia, nausea and vomiting, or anaemia, and the patient went off trial; if valproic acid was responsible, this drug was stopped and VAC continued. If the plasma level of valproic acid was outside the therapeutic range, oral doses were adapted in order to obtain concentration between 50 and $100 \mu \mathrm{g} \cdot \mathrm{mL}^{-1}$ without CT delay. In cases of overdose, valproic acid was stopped and reintroduced only if all adverse events had resolved.

Response was evaluated every three cycles according to WHO criteria. Early cancer progression and stopping treatment due to excessive toxicity were considered treatment failures. Response status was assessed during regular meetings of the groups by at least three independent observers. Survival and PFS were measured from the first day of registration until death (or the last date the patient was known to be alive) or date of first progression or death, respectively. Toxicity was evaluated according to WHO criteria.

\section{Statistical considerations}

The primary end-point was PFS rate at 6 months in order to assess whether the combined treatment was potentially active enough to be further studied. The sample size evaluation relied on the following assumptions. From previous trials [13-15], it was expected to reach a median PFS and 6-month PFS rate of 2.4 months and $18 \%$, respectively, with second-line CT in refractory SCLC patients. It appeared reasonable to expect an increase of the 6-month PFS rate to 39\% (hazard ratio 0.55) with valproic acid and VAC. If true, this increase should be detected by the present trial with $\alpha=10 \%$ and $\beta=10 \%$ after inclusion of 43 patients assessable for PFS.

\section{Results}

Between November 2008 and December 2013, 64 patients were registered, of whom six were not eligible, either because no evidence of progression was documented at time of inclusion $(n=4)$, or because of reduced LVEF or mixed histology (both $\mathrm{n}=1$ ). The main characteristics of the 58 eligible patients are presented in table 1 . 


\section{TABLE 1 Main patient characteristics of the 58 eligible patients included in the study}

\begin{tabular}{lc} 
Age years median (range) & 60 (32-81) \\
Sex & 38 \\
$\quad$ Male & 20 \\
Female & 4 \\
Karnofsky performance status & 13 \\
$\quad 60$ & 18 \\
70 & 13 \\
80 & 10 \\
90 & \\
100 & 38 \\
Previous lines of CT & 19 \\
1 & 37 \\
$>1$ & 14 \\
Free interval after last CT & 7 \\
$<3$ months & 3 \\
$>3$ months & \\
Missing & \\
\hline Data are presented as $n$ unless otherwise stated. CT: chemotherapy. & \\
\hline
\end{tabular}

Seven patients did not receive any treatment and were excluded from the analyses, five because of rapid deterioration due to lung cancer and the cause of death of the last two patients was unknown. The number of CT cycles administered is reported in table 2. Valproic acid dosages were assessed regularly in order to maintain the blood concentration between 50 and $100 \mu \mathrm{g} \cdot \mathrm{mL}^{-1}$. Of the 48 patients with valproic acid data available, 19 had at least one cycle with at least one measurement $<50 \mu \mathrm{g} \cdot \mathrm{mL}^{-1}$ and eight had at least one cycle with at least one measurement $>100 \mu \mathrm{g} \cdot \mathrm{mL}^{-1}$. If we consider all valproic acid data (all cycles, $\mathrm{n}=206$ ), we found that doses $<50$ or $>100 \mu \mathrm{g} \cdot \mathrm{mL}^{-1}$ were found in $19 \%$ and $6 \%$ of the cases, respectively.

Best response statuses are shown in table 3 . Including the 51 patients receiving at least one cycle of CT, the RR was $19.6 \%$ (95\% CI 8.7-30.5\%) and disease control rate was $29 \%$ (95\% CI $19 \%-43 \%)$. Of the 15 patients reaching a partial response or stable disease after three CT cycles, 10 progressed at six cycles.

The median and 6-month PFS were 2.59 months (95\% CI 2.39-2.85 months) and 5\% (SE 3\%) when considering the 58 eligible patients (intent-to-treat analysis). When restricting the analysis to the 51 patients receiving at least one CT injection, median and 6-month PFS times were 2.75 months (95\% CI 2.46-3.61 months) and 6\% (SE 3\%) (figure 1). No statistical difference was found between patients with recurrence $>3$ months after the last $\mathrm{CT}$ and those with a time interval $<3$ months $(\mathrm{p}=0.52)$.

Among the 58 eligible patients, MST was 5.3 months (95\% CI 3.8-7.1 months). At 6, 9 and 12 months, survival rates were $44 \%$ (SE 7\%), 23\% (SE 6\%) and 5\% (SE 4\%). Of the 51 patients receiving CT, 48 died within 6 months after the date of registration. MST was 5.9 months (95\% CI 4.7-7.5 months). At 6, 9 and 12 months, survival rates were 50\% (SE 7\%), 26\% (SE 6\%) and 6\% (SE 4\%). In addition, no significant difference was found according to delay from the last $\mathrm{CT}$ ( $>3$ versus $<3$ months) $(\mathrm{p}=0.45)$.

\section{TABLE 2 Number of chemotherapy cycles administered to the 58 eligible patients}

\section{Cycles}

Patients 
TABLE 3 Best response observed among the 51 eligible patients receiving VAC plus valproic acid in refractory/relapsing small cell lung cancer

Type of response

Subjects $n$

Complete response

Partial response

No change

5

Progression

Early death due to cancer

Early death due to toxicity

High toxicity

Not evaluable

VAC: doxorubicin, vindesine and cyclophosphamide.

As expected, toxicity was mainly haematological (table 4). Only one toxic death (septic shock in febrile neutropenia) was observed. Treatment has to be stopped for excessive toxicity in two cases because of hyperammonaemia related to carbonic anhydrase Va deficiency in one and alteration of the general status of the other, possibly due to chemotherapy.

\section{Discussion}

This phase II study assessing the additive role of an epigenetic modulation by valproic acid to a conventional chemotherapy regimen including doxorubicin, cyclophosphamide and vindesine did not reach its primary objective of improving 6-month PFS despite an interesting RR and 6-month overall survival.

The efficacy of conventional CT has reached a plateau for both SCLC and non-small cell lung cancer (NSCLC). In NSCLC, major therapeutic advances have been noted with targeted therapies, mainly focusing on EGFR activating mutations [16] and ALK translocations [17], or with active immunotherapy [18]. Although a few potential targets have been discovered in SCLC, these have not yet led to therapeutic achievements. This justifies the search for original concepts using old drugs with well-known therapeutic profiles, such as valproic acid.

Valproic acid belongs to the family of HDAC inhibitors. It has major advantages: it has been used for treating epilepsy for more than 40 years, and its pharmacology and toxicology are well characterised; and among classic antiepileptic drugs, it has the lowest interaction with CT [19]. Valproic acid showed anticancer activity in various haematological and solid tumour cell lines, and in mouse models. For example, valproic acid improved the anticancer activity of cisplatin and etoposide in SCLC cell lines [20]. HDAC inhibitors have mainly been tested in haematological malignancies, with some positive results [21]. A few phase I studies have been performed in solid tumours [22-25]. In all but one study, valproic acid was given over a short period of time at a high dose and, consequently, with substantial neurotoxicity.

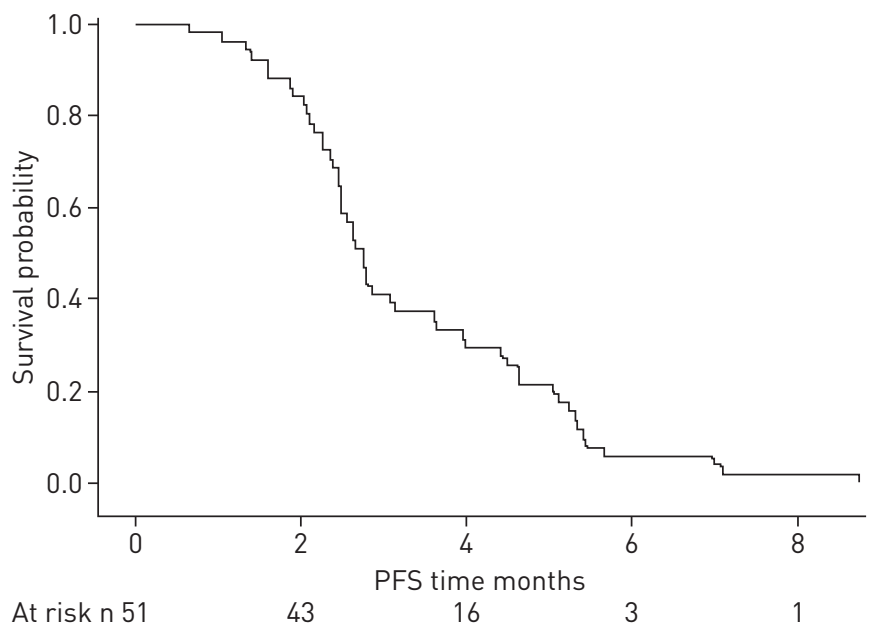

FIGURE 1 Progression-free survival (PFS) time in the 51 patients receiving at least one cycle of chemotherapy. Median PFS was 2.75 months (95\% Cl 2.46-3.61 months) and 6-month PFS was 6\% (SE 3\%). 


\begin{tabular}{|c|c|}
\hline \multicolumn{2}{|l|}{ Nonhaematological toxicity } \\
\hline Nausea and vomiting & $1(2)$ \\
\hline Infection & $2(4)$ \\
\hline Bleeding & $1(2)$ \\
\hline Neurological encephalopathy & $2(4)$ \\
\hline Respiratory & $3(7)$ \\
\hline Cardiac & $1(2)$ \\
\hline Febrile neutropenia & $13(26)$ \\
\hline \multicolumn{2}{|l|}{ Haematological toxicity } \\
\hline Leukopenia & 43 (86) \\
\hline Thrombopenia & $13(26)$ \\
\hline Neutropenia & $44(88)$ \\
\hline
\end{tabular}

Nevertheless, our group was able to demonstrate the value of an epigenetic modulation by valproic acid in malignant pleural mesothelioma [26]. In heavily pre-treated patients, a combination of doxorubicin and valproic acid showed an interesting $16 \%$ response rate, while two previous studies demonstrated that doxorubicin alone was inactive in this setting.

Despite all this evidence, the addition of valproic acid to VAC did not result in improved PFS, the primary end-point of the present study. However, the RR (19.6\%) and median overall survival (5.3 months) were comparable to those reported in a recent systematic review in resistant/refractory SCLC: $14.8 \%$ and 5.45 months, respectively [7]. If our results in terms of PFS and MST appear worse than in previous randomised trials that also included sensitive and refractory SCLC (table 5), it must be taken into consideration that our study included tumours refractory to platinum-etoposide, which have an intrinsically poorer prognosis.

Do we have any explanation for the failure of this approach in SCLC, in contrast to mesothelioma? We can consider various possibilities. First, relapsing SCLC could be very resistant tumours, particularly in the present study, where sensitive tumours to first-line CT were excluded, and that modulating gene expression cannot be sufficient to restore chemosensitivity. Despite very interesting preclinical data [20], it remains difficult to extrapolate from results obtained in cell lines to humans in the clinic. It could be hypothesised that HDACs involved in gene regulation in relapsed SCLC are not those modulated by valproic acid. Another option is that the dosage of valproic acid chosen in this study, corresponding to the

TABLE 5 Response rate (RR), progression-free survival (PFS) and survival in randomised trials with salvage chemotherapy for relapsing small cell lung cancer

\begin{tabular}{|c|c|c|c|c|c|c|}
\hline $\begin{array}{l}\text { First author } \\
\text { [ref.] }\end{array}$ & Regimen & $\begin{array}{l}\mathbf{R R} \\
\%\end{array}$ & PFS & MST & $\begin{array}{c}\text { 6-month survival } \\
\%\end{array}$ & $\begin{array}{c}1 \text {-year survival } \\
\%\end{array}$ \\
\hline \multirow[t]{2}{*}{ Von Pawel [8] } & VAC & 18 & 12.3 weeks & 25 weeks & 45 & 14.4 \\
\hline & Topotecan & 24 & 13.3 weeks & 25 weeks & 47 & 14.2 \\
\hline \multirow[t]{2}{*}{ O'BRIEN [4] } & Topotecan & 7 & 16.3 weeks & 25.9 weeks & 49 & \\
\hline & $\mathrm{BSC}$ & & & 13.9 weeks & 26 & \\
\hline \multirow[t]{2}{*}{ ECKARDT [9] } & $\begin{array}{l}\text { Topotecan } \\
\text { oral }\end{array}$ & 18 & 11.9 weeks & 33 weeks & & 32.6 \\
\hline & Topotecan IV & 22 & 14.6 weeks & 35 weeks & & 29.2 \\
\hline \multirow[t]{2}{*}{ INOUE [27] } & Amrubicin & 38 & 3.5 months & 8.1 months & & \\
\hline & Topotecan & 13 & 2.2 months & 8.4 months & & \\
\hline \multirow[t]{2}{*}{ JotTe [28] } & Amrubicin & 44 & 4.5 months & 9.2 months & 60 & 36 \\
\hline & Topotecan & 15 & 3.3 months & 7.6 months & 54 & 33 \\
\hline $\begin{array}{l}\text { Present } \\
\text { study }\end{array}$ & VAC-VA & 20 & 2.6 months & 5.3 months & 50 & 6 \\
\hline
\end{tabular}

MST: median survival time; VAC: doxorubicin, vindesine and cyclophosphamide; BSC: best supportive care; VA: valproic acid. 
one used for treating epilepsy, was below the concentration needed to obtain HDAC inhibition and that the $1 \mathrm{mM}$ used in cell lines was higher than the one obtained in clinical practice [20]. In addition, we observed that in $19 \%$ of the measurements, valproic acid dosages were below the recommended value at least once during CT. However, as this is not uniform throughout all cycles, it seems unlikely that low dosages of valproic acid could be implicated in the limited efficacy of VAC.

A last concern could be the choice of vindesine instead of vincristine in the triplet combination. Vindesine is commonly used in SCLC. The ELCWP conducted randomised trials in first-line therapy comparing etoposide plus vindesine with and without cisplatin [29], assessing the role of accelerated CT (vindesine, ifosfamide and epirubicin) [30] with maintenance therapy with etoposide and vindesine [31]. In the second line, vindesine showed activity when added to cisplatin for refractory [32] or recurrent SCLC [33]. In all these studies, toxicity, particularly neurotoxicity, was manageable. In the few trials comparing vindesine to vincristine, including a randomised trial in combination with cyclophosphamide as first-line chemotherapy in 116 patients with SCLC [34], similar toxicity and efficacy were noted.

In conclusion, epigenetic modulation with valproic acid failed to improve the therapeutic efficacy of a conventional CT regimen for relapsed refractory SCLC, preventing further evaluation of this combination. However, as preclinical data are showing promising results, more biological information is needed in order to pursue research in this field.

\section{Acknowledgements}

The participating institutions were: Institut Jules Bordet, Brussels, Belgium (J-P. Sculier, T. Berghmans, A-P. Meert, M. Paesmans, E. Markiewicz, P. Van Houtte, M. Roelandts, N. Leclercq and I. CsToth); Hôpital Saint-Joseph, Gilly, Belgium (B. Colinet); Hôpital Erasme, Brussels, Belgium (T. Berghmans and Z. Mekinda); Hôpital Ambroise Paré, Mons, Belgium (P. Wackenier and S. Holbrechts); CHU de Charleroi, Charleroi, Belgium (J. Lecomte); Centre Hospitalier de Mouscron, Mouscron, Belgium (C. Tulippe); CH Peltzer-La Tourelle, Verviers, Belgium (Y. Bonduelle); CHRU Calmette, Lille, France ( J-J. Lafitte, A. Scherpereel and A. Cortot).

\section{References}

1 Houston KA, Henley SJ, Li J, et al. Patterns in lung cancer incidence rates and trends by histologic type in the United States, 2004-2009. Lung Cancer 2014; 86: 22-28.

2 Mascaux C, Paesmans M, Berghmans T, et al. A systematic review of the role of etoposide and cisplatin in the chemotherapy of small cell lung cancer with methodology assessment and meta-analysis. Lung Cancer 2000; 30: 23-36.

3 Pujol JL, Carestia L, Daures JP. Is there a case for cisplatin in the treatment of small-cell lung cancer? A meta-analysis of randomized trials of a cisplatin-containing regimen versus a regimen without this alkylating agent. Br J Cancer 2000; 83: 8-15.

4 O'Brien ME, Ciuleanu TE, Tsekov H, et al. Phase III trial comparing supportive care alone with supportive care with oral topotecan in patients with relapsed small-cell lung cancer. J Clin Oncol 2006; 24: 5441-5447.

5 Pelayo AM, Westeel V, Cortes-Jofre M, et al. Chemotherapy versus best supportive care for extensive small cell lung cancer. Cochrane Database Syst Rev 2013; 11: CD001990.

6 Riemsma R, Simons JP, Bashir Z, et al. Systematic review of topotecan (Hycamtin) in relapsed small cell lung cancer. BMC Cancer 2010; 10: 436.

7 Owonikoko TK, Behera M, Chen Z, et al. A systematic analysis of efficacy of second-line chemotherapy in sensitive and refractory small-cell lung cancer. J Thorac Oncol 2012; 7: 866-872.

8 von Pawel J, Schiller JH, Shepherd FA, et al. Topotecan versus cyclophosphamide, doxorubicin, and vincristine for the treatment of recurrent small-cell lung cancer. J Clin Oncol 1999; 17: 658-667.

9 Eckardt JR, von Pawel J, Pujol JL, et al. Phase III study of oral compared with intravenous topotecan as second-line therapy in small-cell lung cancer. J Clin Oncol 2007; 25: 2086-2092.

10 von Pawel J, Gatzemeier U, Pujol JL, et al. Phase ii comparator study of oral versus intravenous topotecan in patients with chemosensitive small-cell lung cancer. J Clin Oncol 2001; 19: 1743-1749.

11 Scherpereel A, Gentina T, Grigoriu B, et al. Overexpression of endocan induces tumor formation. Cancer Res 2003; 63: 6084-6089.

12 Hubaux R, Vandermeers F, Cosse J-P, et al. Valproic acid improves second-line regimen of small cell lung cancer in preclinical models. ERJ Open Res 2015; 1: 00028-2015.

13 Ardizzoni A, Manegold C, Debruyne C, et al. European organization for research and treatment of cancer (EORTC) 08957 phase II study of topotecan in combination with cisplatin as second-line treatment of refractory and sensitive small cell lung cancer. Clin Cancer Res 2003; 9: 143-150.

14 Christodoulou C, Kalofonos HP, Briasoulis E, et al. Combination of topotecan and cisplatin in relapsed patients with small cell lung cancer: a phase II study of the Hellenic Cooperative Oncology Group (HeCOG). Cancer Chemother Pharmacol 2006; 57: 207-212.

15 Rocha-Lima CM, Herndon JE, Lee ME, et al. Phase II trial of irinotecan/gemcitabine as second-line therapy for relapsed and refractory small-cell lung cancer: Cancer and Leukemia Group B Study 39902. Ann Oncol 2007; 18: 331-337.

16 Gao G, Ren S, Li A, et al. Epidermal growth factor receptor-tyrosine kinase inhibitor therapy is effective as first-line treatment of advanced non-small-cell lung cancer with mutated EGFR: a meta-analysis from six phase III randomized controlled trials. Int J Cancer 2012; 131: E822-E829.

17 Shaw AT, Kim DW, Nakagawa K, et al. Crizotinib versus chemotherapy in advanced ALK-positive lung cancer. N Engl J Med 2013; 368: 2385-2394. 
Brahmer JR, Tykodi SS, Chow LQ, et al. Safety and activity of anti-PD-L1 antibody in patients with advanced cancer. N Engl J Med 2012; 366: 2455-2465.

19 Vecht CJ, Wagner GL, Wilms EB. Interactions between antiepileptic and chemotherapeutic drugs. Lancet Neurol 2003; 2: 404-409.

20 Hubaux R, Vandermeers F, Crisanti MC, et al. Preclinical evidence for a beneficial impact of valproate on the response of small cell lung cancer to first-line chemotherapy. Eur J Cancer 2010; 46: 1724-1734.

21 Stimson L, Wood V, Khan O, et al. HDAC inhibitor-based therapies and haematological malignancy. Ann Oncol 2009; 20: 1293-1302.

22 Munster P, Marchion D, Bicaku E, et al. Phase I trial of histone deacetylase inhibition by valproic acid followed by the topoisomerase II inhibitor epirubicin in advanced solid tumors: a clinical and translational study. J Clin Oncol 2007; 25: 1979-1985.

23 Chavez-Blanco A, Segura-Pacheco B, Perez-Cardenas E, et al. Histone acetylation and histone deacetylase activity of magnesium valproate in tumor and peripheral blood of patients with cervical cancer. A phase I study. Mol Cancer 2005; 4: 22.

24 Atmaca A, Al-Batran SE, Maurer A, et al. Valproic acid (VPA) in patients with refractory advanced cancer: a dose escalating phase I clinical trial. Br J Cancer 2007; 97: 177-182.

25 Chu BF, Karpenko MJ, Liu Z, et al. Phase I study of 5-aza-2'-deoxycytidine in combination with valproic acid in non-small-cell lung cancer. Cancer Chemother Pharmacol 2013; 71: 115-121.

26 Scherpereel A, Berghmans T, Lafitte JJ, et al. Valproate-doxorubicin: promising therapy for progressing mesothelioma. A phase II study. Eur Respir J 2011; 37: 129-135.

27 Inoue A, Sugawara S, Yamazaki K, et al. Randomized phase II trial comparing amrubicin with topotecan in patients with previously treated small-cell lung cancer: North Japan Lung Cancer Study Group Trial 0402. J Clin Oncol 2008; 26: 5401-5406.

28 Jotte R, Conkling P, Reynolds C, et al. Randomized phase II trial of single-agent amrubicin or topotecan as second-line treatment in patients with small-cell lung cancer sensitive to first-line platinum-based chemotherapy. J Clin Oncol 2011; 29: 287-293.

29 Sculier JP, Klastersky J, Libert P, et al. A randomized study comparing etoposide and vindesine with or without cisplatin as induction therapy for small cell lung cancer. EORTC Lung Cancer Working Party. Ann Oncol 1990; 1: 128-133.

30 Sculier JP, Paesmans M, Lecomte J, et al. A three-arm phase III randomised trial assessing, in patients with extensive-disease small-cell lung cancer, accelerated chemotherapy with support of haematological growth factor or oral antibiotics. Br J Cancer 2001; 85: 1444-1451.

31 Sculier JP, Paesmans M, Bureau G, et al. Randomized trial comparing induction chemotherapy versus induction chemotherapy followed by maintenance chemotherapy in small-cell lung cancer. European Lung Cancer Working Party. J Clin Oncol 1996; 14: 2337-2344.

32 Chiuten DF, Carr DT, Dhingra HM, et al. Combination chemotherapy with vindesine and cisplatin for refractory small cell bronchogenic carcinoma. Cancer Treat Rep 1986; 70: 795-796.

33 Niederle N, Schutte J, Schmidt CG, et al. Treatment of recurrent small cell lung carcinoma with vindesine and cisplatin. Cancer Treat Rep 1984; 68: 791-792.

34 Niiranen A, Holsti LR, Salmo M, et al. Vincristine-cyclophosphamide, the classical two-drug regimen for small-cell lung cancer, evaluated in a randomized study with vindesine. Am J Clin Oncol 1987; 10: 507-511. 